\title{
The Importance of the Nurse's Role in the Management of Complex Diabetic Outpatients: It is the Time to Manage Patient's Multidimensions
}

\author{
Marika Lo Monaco ${ }^{2,3,4}$, Raffaella Mallaci Bocchio ${ }^{2,3}$, Giuseppe Natolii ${ }^{2,3}$, Salvatore Corrao*1,2,3,4 \\ ${ }^{1}$ Department of internal medicine and medical specialities, Centre of Research for Effectiveness and Appropriateness in Medicine, Italy \\ ${ }^{2}$ National Relevance and High Specialization Hospital Trust ARNAS Civico, Di Cristina,Benfratelli in Palermo \\ ${ }^{3}$ Department of Science and Technology, Euro Mediterranea Institute of Science and Technology
}

${ }^{4}$ Doctorate School of biomedicine and Neuroscience University of Palermo, Italy

Received: June 01, 2018; Published: June 12, 2018

*Corresponding author: Salvatore Corrao, Internal Medicine Department, Italy

Keywords: Nursing Assessment; Diabetes Mellitus; Comorbidity; Outpatients; Clinical pathway; Patient Care Management; Chronic Disease; Needs Assessment; Clinical Complexity; Therapeutic Reconciliation; Diabetes Quality of life

Abbreviations: DDI: Drug-Drug Interactions; CIRS: Cumulative Illness Rating Scale; DQOL: Diabetes Quality of life; ABI: Anklebrachial Index; MMSE: Mini-Mental State Examination; SBT: Short Blessed Test; GDS : Geriatric Depression Scale; MNA: Mini Nutritional Assessment Test

\section{Introduction}

Diabetes is a significant chronic disease, 424.9 million people worldwide are estimated to have diabetes in 2017, and this number is expected to increase to 628.6 million by 2045 [1]. More and more patients with diabetes are affected by other comorbidities, in fact, more than $40 \%$ of diabetic people have 3 or more comorbidities [2]. Another important aspect is that, as population ageing, health needs tend to become more complex, multimorbidity, the co-occurrence of multiple diseases in the same patient, represents the most relevant feature among older adults, with a prevalence from $55 \%$ to $98 \%$ [3]. Inevitably, the coexistence of chronic diseases is often accompanied by poly pharmacy (daily intake of five or more drugs), that could create a frailty condition, poor adherence to therapies, unknown Diabetes Quality of life (DDI) and inappropriate hospital admission, with significant implications on economic resources [4, 5]. In light of these reasons the management of complex diabetic outpatients should be reviewed, and nurses might play a central role to improve quality of care and patient quality of life.

This paper aims to describe our new model to assess complex outpatients affected by diabetes mellitus. In an internal medicine outpatient clinic at the National Relevance and High Specialization Hospital Trust ARNAS Civico in Palermo (Italy), a specific pathway to manage complex diabetic outpatients based on the multidimensional assessment with strong nurse coordination was set up in 2016 and is described as follow. According to the model a nurse manage the entire process and each outpatient is evaluated for specific personal, anamnestic and clinical data and receive educational advice by a multi-professional team made of specialised physicians, a coordinating nurse, a nutritionist, a podiatrist and a clinical pharmacologist. At admission, each outpatient does the first clinical evaluation with the specialised physician who collects the clinical history also using the Cumulative Illness Rating Scale (CIRS) and does the physical examination. Each patient receives a complete cardiovascular, respiratory and metabolic assessment. The nurse assesses patients' vital signs, quality-of-life using the Diabetes Quality of life (DQOL) and potential peripheral artery disease through the Ankle Brachial Index (ABI). Moreover, the nurse assesses cognitive function by the Mini-Mental State Examination (MMSE) or the Short Blessed Test (SBT), this last in the elderly patients. In the same way, the nurse uses Geriatric depression scale (GDS) and the Hamilton rating scale for depression. Finally, patient autonomy is evaluated with the Barthel Index.

The nutritional assessment includes Body mass index calculation, Waist circumference evaluation and the nurse's role in 
the management of complex diabetic outpatients. Mini nutritional assessment test (MNA), body composition analysis by bioelectrical impedance, and heel ultrasound scan. All our outpatients with diabetes receive nurse education about lifestyles and the use of diabetic devices [6], specific nutritional advises, and podiatrist education on foot care. The coordinating physician provides diagnostic and therapeutic reconciliation [7]. The co-ordinating nurse takes care of the entire patient pathway and evaluates patient needs and caregiver support. Moreover, the clinical pharmacologist evaluates in every phase of drug prescription the appropriate treatments (through the use of the Beers criteria, START and STOPP ones) and early identifies drug-drug interactions (through the use of specific databases, e.g., Micromedex) [8]. At discharge, a detailed medical and nurse report is sent to the general practitioner for the best patient follow up. However, the hospital nurse coordinates early admissions of each patient in the case of clinical instability by direct links with the general practitioner network and periodic telephone calls to, particularly complex patients.

\section{Conclusion}

The implementation of the multidimensional assessment of patients with diabetes coordinated by the nurse improves diabetes' management and allows clinicians to have a whole vision of the complexity to better assess patients affected by multiple chronic conditions. That complex pathway could represent in the future a new prerogative to improve patients' self-management and therapeutic adherence for all their comorbidities reducing inappropriate hospital admissions and healthcare costs [9].

\section{References}

1. (2017) International Diabetes Federation. IDF Diabetes Atlas, ( $8^{\text {th }}$ edn, $)$. International Diabetes Federation Brussels, Belgium.

2. Sinnige J, Braspenning J, Schellevis F, Stirbu Wagner I, Westert G, et al. (2013) The prevalence of disease clusters in older adults with multiple chronic diseases: A systematic literature review. Plosone 8(11): 79641.

3. Vetrano D, Calderon Larranaga A, Marengoni A, Onder G, Bauer JM, et al. (2017) An International Perspective on Chronic Multimorbidity: Approaching the Elephant in the Room. J Gerontol A Biol Sci Med Sci.

4. Doucet J, Chassagne P, Trivalle C, Landrin I, Pauty, et al. (1996) Drug drug interactions related to hospital admissions in older adults: prospective study of 1000 patients. J Am Geriatr Soc 44(8): 944-948.

5. Lindley CM, Tully MP, Paramsothy V, Tallis RC (1992) Inappropriate medication is a major cause of adverse drug reactions in elderly patients. Age Ageing 21(4): 294-300.

6. Dudley JD (1980) The diabetes educator's role in teaching the diabetic patient. Diabetes Care 3(1): 127-133.

7. Pandolfe F, Wright A, Slack WV, Safran C (2018) Rethinking the outpatient medication list: increasing patient activation and education while architecting for centralization and improved medication reconciliation. J Am Med Inform Assoc.

8. Choi S, Babiak J (2018) Evaluation of Pharmacist-Initiated Discharge Medication Reconciliation and Patient Counseling Procedures. Consult Pharm 33(4): 222-226.

9. Introduction (2018) Standards of Medical Care in Diabetes. Diabetes Care 41(1).
(C) (P) This work is licensed under Creative

Submission Link: https://biomedres.us/submit-manuscript.php

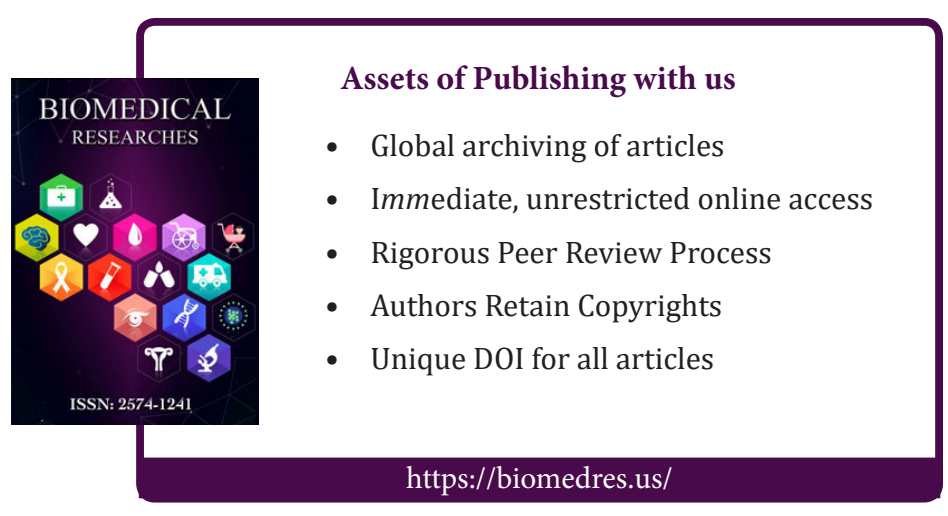

